\title{
THE IDENTIFICATION OF EAR PRINTS USING COMPLEX GABOR FILTERS
}

\author{
Alexander A S Gunawan; Heni Kurniaty; Wikaria Gazali \\ Mathematics and Statistics Department, School of Computer Science, Bina Nusantara University \\ Jl. KH. Syahdan No. 9, Jakarta 11480, Indonesia \\ aagung@binus.edu; wikaria@binus.edu
}

\begin{abstract}
Biometrics is a method used to recognize humans based on one or a few characteristics physical or behavioral traits that are unique such as DNA, face, fingerprints, gait, iris, palm, retina, signature and sound. Although the facts that ear prints are found in $15 \%$ of crime scenes, ear prints research has been very limited since the success of fingerprints modality. The advantage of the use of ear prints, as forensic evidence, are it relatively unchanged due to increased age and have fewer variations than faces with expression variation and orientation. In this research, complex Gabor filters is used to extract the ear prints feature based on texture segmentation. Principal component analysis (PCA) is then used for dimensionality-reduction where variation in the dataset is preserved. The classification is done in a lower dimension space defined by principal components based on Euclidean distance. In experiments, it is used left and right ear prints of ten respondents and in average, the successful recognition rate is $78 \%$. Based on the experiment results, it is concluded that ear prints is suitable as forensic evidence mainly when combined with other biometric modalities.
\end{abstract}

Keywords: Biometrics; Ear prints; Complex Gabor filters; Principal component analysis; Euclidean distance

\section{INTRODUCTION}

Surveillance technologies now widely used by the society. The very fast growth of security cameras is caused by the numbers of increasing crimes, and also the environmental needs that are safe but lack of labors to oversee the neighborhood. The non-invasive biometric technology users are the important since they improve the usability of the security cameras by building automated visual surveillance systems. Non-invasive biometric technologies is the example of the improvement by building the facial recognition and the ear prints that are useful for forensic investigation based on the camera records. Therefore, nowadays, criminals are familiar with disguises to hide their identities, so the facial recognition becomes less effective for this forensic investigation needs. Here, the ear prints recognition can be the alternative way that is promising. For example, it can be seen in Fig. 1, the suspect uses the pet cap to hide his identity. However, his ear can be clearly seen, although with a low resolution. Notice that the disguise, that is used to avoid the identification, can be socially accepted.

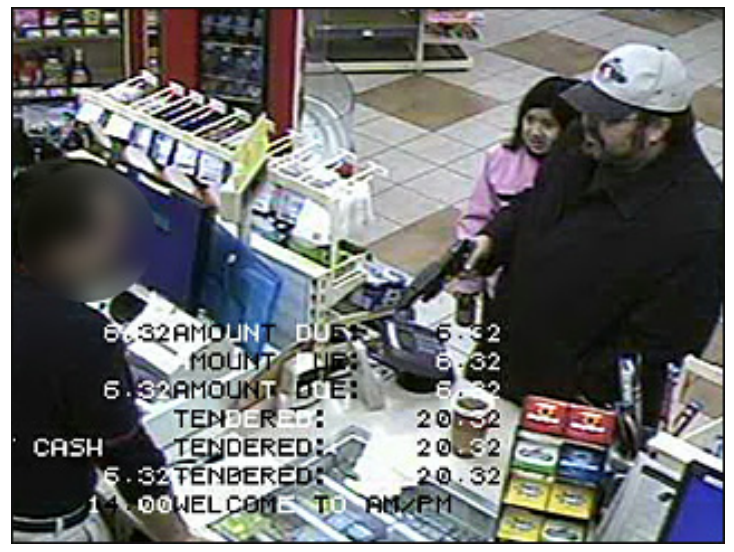

Fig. 1: An example of the use of ear prints for the forensic investigations

Human ears are the very stable structures, which are rich in information and easily imaged for biometric identifications. The biometric of ear prints is often compared with the biometric of face [1]. Ear has several excellences rather compared face, especially because ear is relatively unchanged due to increased age, colors of skin, and hair styles [2]. It has fewer variations than faces with expressions variations and orientations [3]. The uniqueness of the form of ear to any human being is useful for forensic investigation, because it is a 
part of the body that is rarely of hardly noticed. The ear print has been used in the forensic science for a long time [4]. If it is compared with other biometrics, such as iris, retina, and fingerprint, the ear remains greater and easier to be apprehended at a distance by using a camera. Besides for forensic investigation, ear actually is also a part of disaster identification system. By using the biometric of ear, disaster victims can be identified although the face cannot be recognized anymore.

Next, the orders of writing here are organized as follows: Firstly, the explanation about ear and the identification system of ear prints. Then, it continues with the explanation about complex Gabor filters and its use in the extraction of ear features based on segmentation texture. And it is continued with the description about principal component analysis (PCA) to reduce the dimension of features where the variation on its image remains maintained. The classification is done in the lower dimension room that is defined by the PCA based on Euclidean distance. Lastly, it will be described about the experiment that is conducted, along with the conclusion that is obtained.

\section{METHOD}

The use of ear as the modality in biometrics has been done long, at least a hundred year ago [4]. However until now, still a question emerges whether that ear is unique to each individual. The structure of ear in Figure 1, it is quite complex, so the possibility of the structure of ears, that is exactly the same from two individuals, is very small. The most famous research in the field of ear identifications was made by Alfred Iannarelli in 1989, when he collected more than ten thousand ears and found that all of them were different [2]. In 1906, Imhofer found that from five hundred data of ears, only four characteristics are needed to determine the ear which is unique [5].

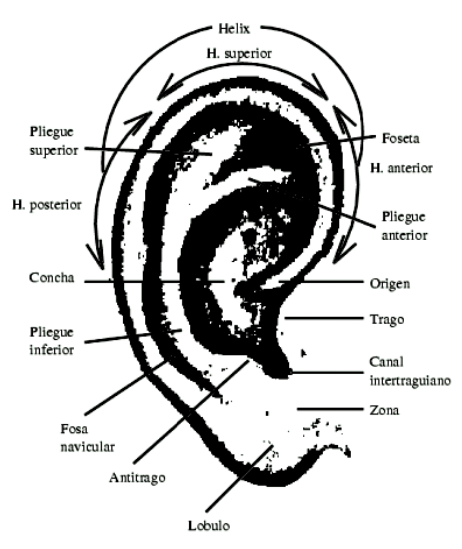

Fig 1: The Structure of ear [6]
A specialist company of Bromba $\mathrm{GmbH}$ biometric (2003) has compared a different variety of biometrics, including the form of ear especially from the stability aspect against some variables, which are: growth, aging, dung, and injury. A good biometric will have a high constancy, so there is only a few perhaps variation, although it is in the influence of the variables. Based on this research, the biometric of ear is more permanent than the biometrics of face, signature, and voice. The biometric of ear is only less permanent than the biometric that is invasive like DNA and iris. Even though there is a fact that ear prints have been found fifteen percent from the crime scene, the research of ear prints is very limited because the success of the research from fingerprints. With the previous reasons, we are doing a research about ear prints identifications.

The system of ear identification generally includes the main module, as follows: detection, pre-processing, extraction feature, and earmatching (Fig. 2). The detection of the ear in an image is the early step to do the ear identification. By detecting the ear, it will be obtained the image of the form of ear that fit to be identified. In this research, the Viola-Jones method is used to detect the ear. The next step is the pre-processing by doing a normalization of the image size, a conversion into grayscale image, and an improvement of the light intensity with histogram equalization method. Next, it is done an extraction feature by using the complex Gabor filters, so that the structure of the ear can be made contrast based on the segmentation of the texture. The last step is the ear-matching by using the principal component analysis (PCA) that is used to reduce the dimension features where the variation on its image remains maintained. Next, a classification is done in the lower dimension room that is defined by the PCA based on the Euclidean distance.

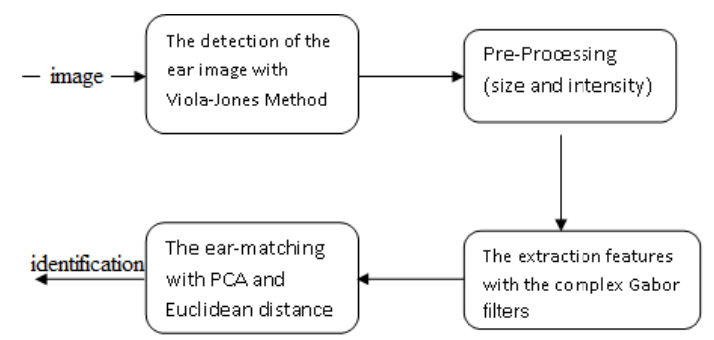

Fig. 2: The process of ear identification

\section{Viola Jones Method}

The first step is the ear detection using Viola Jones method. Viola Jones method is the algorithm of objects detections that are often used to detect objects. This is because this method has the algorithm that is strong (robust) and efficient. 
The process of object detection in an image using Viola Jones method is done, after the learning process, to obtain the classification that is formed from the data training. There are four principle stages in Viola Jones method [7], which are: (1) The Haar-like extraction features. (2) The making of integral image. (3) the learning features with Adaptive Boosting (AdaBoost). (4) The compound classification with the Cascade of Classifier.

\section{Pre-Processing}

With the size of different images, it will be very difficult for us to compare the features in the image. Then, a process to normalize the size of the image is needed. Next, to reduce the differences of the illumination and the impacts from disturbance (noise) in the image is by changing it into the grayscale image, and using the histogram equalization method. The purpose of histogram equalization method is to produce a histogram image that is harmonious, so that the image will seem more contrast with the light intensity that is more or less almost the same. This method can be used in the overall image or in some parts of the image only.

\section{Complex Gabor Filters}

The ability of the human visual system in distinguishing various textures is based on the capability in identifying variety of frequencies and spatial orientations from the texture which is being observed. Gabor filter that is first introduced by Dennis Gabor [8] is one of the filters that can simulate the characteristics of human visual system, in isolating certain frequencies and orientations from images. This characteristic make the Gabor filter appropriate for the texture recognition application in an image. Gabor filter can be used to conduct textural image segmentation based on the repeated local pattern in the certain orientations and frequencies.

In spatial, a Gabor function is a sinusoidal, which is being modulated by Gauss function. An impulse response of a two dimensions complex Gabor filter is:

$$
h(x, y)=\frac{1}{2 \pi \sigma_{x} \sigma_{y}} \exp \left\{-\frac{1}{2}\left[\frac{x^{2}}{\sigma_{x}^{2}}+\frac{y^{2}}{\sigma_{y}^{2}}\right]\right\} \exp (j 2 \pi F x)
$$

and can be illustrated as the following:

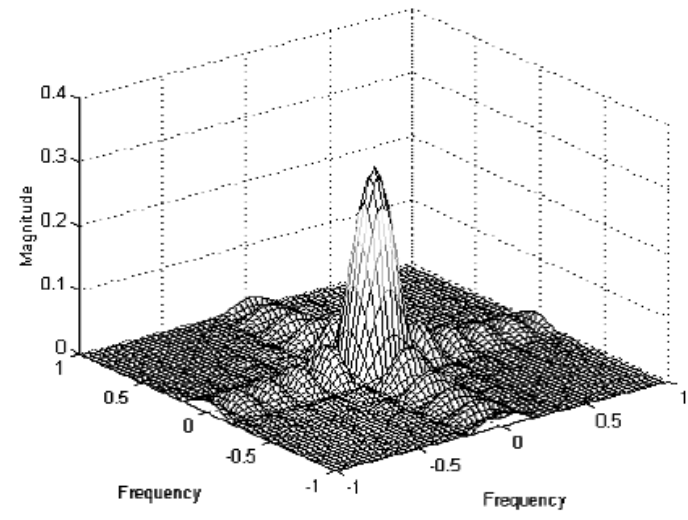

Fig 3: Impulse Response of two dimensions Gabor filter

In the frequency domain, Gabor filter can be represented as the following:

$$
H(u, v)=\exp \left\{-2 \pi^{2}\left\lfloor(u-F)^{2} \sigma_{x}^{2}+v^{2} \sigma_{y}^{2}\right\rfloor\right\}
$$

There are six parameters that should be stipulated in the implementation of Gabor filters. The six parameters are: $F, \theta, \sigma_{x}, \sigma_{y}, B_{F}$, and $B_{\theta}$. Here are the explanations:

Frequency $(F)$ and orientation $(\theta)$ define the central location of the filter. $B_{\varphi}$ and $B_{\theta}$ express the constant band width of frequencies and the angular range of filters.

The variable $\sigma_{x}$ related to the response of $-6 \mathrm{~dB}$ for the spatial frequency component.

$$
\sigma_{x}=\frac{\sqrt{\ln 2\left(2^{B_{F}}+1\right)}}{\sqrt{2} \pi F\left(2^{B_{F}}-1\right)}
$$

The variable $\sigma_{y}$ related to the response of $-6 \mathrm{~dB}$ for the angular component.

$$
\sigma_{y}=\frac{\sqrt{\ln 2}}{\sqrt{2} \pi F \tan \left(B_{\theta} / 2\right)}
$$

The position $(F, \theta)$ and band width $(\sigma, \sigma)$ from Gabor filter in the frequency domain should be meticulously stipulated in order to capture textural information properly.

After obtaining Gabor features, then the extraction feature can be conducted. One of the features, which can be chosen, is the energy feature, that id defined as: 


$$
e(x)=\frac{1}{M N} \sum_{t=1}^{M} \sum_{j=1}^{N}|x(m, n)|^{2}
$$

\section{The principal component analysis \\ and Euclidean distance}

The Principal Component Analysis (PCA) is a method that involves a mathematic procedure to reduce data and extraction feature [9]. This PCA method is projecting the origin space into a new space that has lower dimension, by keeping that as much information as possible which possessed by the origin dimension, are maintained and there are not much that lost after being projected to the new space. By minimizing the dimension from a space, of course it will alleviate the computation process that should be conducted by the system.

Euclidean distance is one of the methods that can be used to do the classification of the image of ear, which is new (unknown), to one of the images of ear which has been known. The weight value between the two vectors $i$ and $j$ that is counted by using the Euclidean distance shows the measures of similarities between the image $i$ and $j$ [10]. The calculation of the Euclidean distance is conducted based on the following formula:

$$
\|x-y\|_{\varepsilon}=\sqrt{\left|x_{i}-y_{i}\right|^{2}}
$$

\section{RESULTS AND DISCUSSION}

The application program of ear prints identification is made by using Microsoft Visual Studio 2010 software, with the programming language $\mathrm{C \#}$ and the library EmguCV. The working mechanism of this application program begins when the user conduct an input by turning on the camera. Next, Viola Jones method will detect whether there is an ear image in every frames in the video that is origin from the camera. If the image of the ear has been detected, then the input of the image will be normalized by the size, be converted into grayscale image, and histogram equalization is conducted. This is for reducing the disturbance (noise) in the image input that causes difficulties in the extraction features of ear. After the image is considered clear from disturbances, then the process of the extraction features with the complex Gabor filter and PCA is begun. In the submenu setting, the appropriate Gabor parameter can be chosen.

In the experiment, data training of the image of ear is taken in a perpendicular manner against the camera. The image that is captured next will go through the processing, as has been explained previously. And last it is given lable based on the respondents' names who are involved. The following Figure 4 is a screen shot from the application program that is developed.

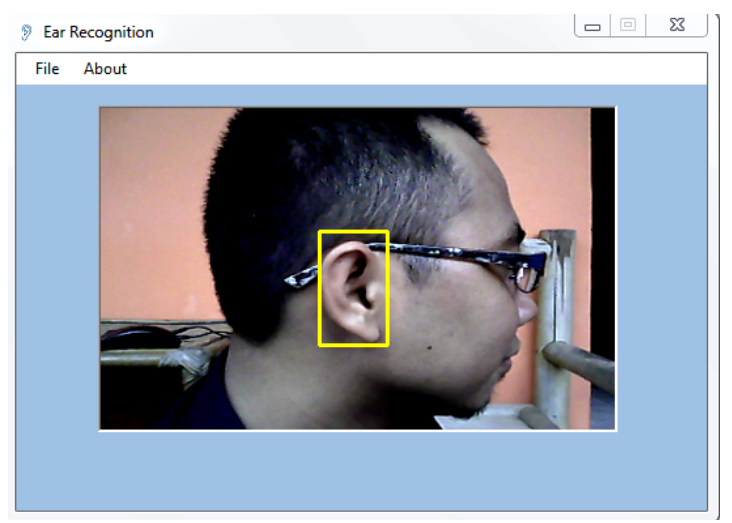

Fig. 4: A Screen shoot of application program

The process of recognition is conducted after the respondents take the image of ear directly with camera as the data training. The detected image of ear is marked by a yellow square. Next, if the image of ear from the respondents is successfully detected, then the process of recognition will be conducted based on the available data training. The application program is conducting recognition by comparing the detected image of ear with the data training which has been kept by using the Euclidean distance. If it is match, in terms of the Euclidean distance, that is calculated, is less than the value of a certain threshold, so the program will release the result of the recognition from the image, like in the Figure 5.

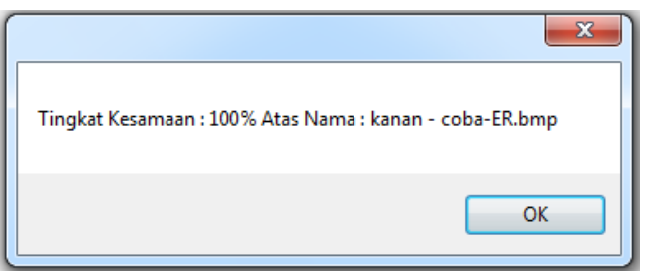

Fig. 5: Image-matching

In testing this application program, ten respondents are incorporated to take the images of the left and the right ears. Each of the respondents do repetitions as many as ten times experiments and then the value of the successful recognitions is taken, as showed in the following Table 1. 
Table 1: The result of the recognition experiment of the left and right ears

\begin{tabular}{ccc}
$\begin{array}{c}\text { Respondent } \\
\text { Number }\end{array}$ & $\begin{array}{c}\text { The percentage of } \\
\text { the success left ear }\end{array}$ & $\begin{array}{c}\text { The percentage of the } \\
\text { success right ear }\end{array}$ \\
\hline $\mathbf{1}$ & $60 \%$ & $70 \%$ \\
$\mathbf{2}$ & $80 \%$ & $90 \%$ \\
$\mathbf{3}$ & $80 \%$ & $70 \%$ \\
$\mathbf{4}$ & $90 \%$ & $70 \%$ \\
$\mathbf{5}$ & $80 \%$ & $60 \%$ \\
$\mathbf{6}$ & $70 \%$ & $70 \%$ \\
$\mathbf{7}$ & $90 \%$ & $90 \%$ \\
$\mathbf{8}$ & $90 \%$ & $80 \%$ \\
$\mathbf{9}$ & $80 \%$ & $80 \%$ \\
$\mathbf{1 0}$ & $80 \%$ & $70 \%$ \\
\hline Average & $\mathbf{8 0} \%$ & $\mathbf{7 5 \%}$ \\
\hline
\end{tabular}

Based on the test result to the reidentification of the image of the left and right ears each as many as ten times experiments, it is obtained the success level of right ear recognition is seventy five percent and the left ear is eighty percent. So, in average the success level of ears recognition is seventy eight percent. This result shows the recognitions of ear prints still cannot be the principal feature for the authentication application, which needs a high accuracy and easy in the detection. But, the ear print is quite adequate, if it is used as an additional feature for the forensic and reconnaissance requirements because it can provide clear hints, besides the ear print is relatively permanent and the image of the ear is also easily obtained.

\section{CONCLUSION}

The application program of the ear identification, by using the complex Gabor filters method for the extraction feature, has a high level of success in identifying the ears of the respondents. This result shows the biometric of ear can be used for forensic investigation and visual surveillance, but it is less suitable to be used for the authentication application, which needs a higher dependability. The main problem in the application of ear identification is in the process of the detection. As for the failure of ear detection is caused by the image of the ear that is too small caught by the camera or experiencing too much deformation of perspective. This detection problem can be solved by doing an increase in the resolution from the area of the targeted image, for example with the super resolution method.

\section{REFERENCES}

[1] A. Bertillon, La Photograhie Judiciaire, avec un appendice sur la classification et l'Identification Anthropometriques. Gauthier-Villars. 1890.

[2] G. Bromba, Bioidentification Frequently Asked Questions. Accessed from http://www.bromba.com/faq/biofaqe.html. 2003.

[3] M. Carreira-Perpinan, (1995). Compression neural networks for feature extraction: Application to human recognition from ear images. Madrid: Technical University of Madrid. 1995.

[4] K. Chang, K. Bowyer, S. Sarkar \& B. Victor, Comparison and combination of ear and face images in appearance-based biometrics. Pattern Analysis and Machine Intelligence, IEEE Transactions, Vol. 25 No. 9, pp. 1160-1165, 2003.

[5] A. Eleyan, H. Demirel, PCA and LDA Based Neural Networks for Human Face Recognition. In K. D. Grgic (Ed.), Face Recognition, 93-106, Intech, 2007.

[6] D. Gabor, Theory of communication. Journal of the Institute of Electrical Engineers, Vol. 93, pp. 429457, 1946.

[7] A. Hoogstrate, H. Van den Heuvel, E. Huyben, Ear Identification Based on Surveillance Camera's Images. Accessed from: http://www.forensicevidence.com/site/ID/IDearCamera.html, 2000, May 31.

[8] A. V. Iannarelli, Ear Identification (Forensic Identification Series). Fremount, California: Paramount Publishing Company, 1989.

[9] H. K. Lammi, Ear Biometrics. Lappeenranta, Finland: Lappeenranta University of Technology, Department of Information Technology, Laboratory of Information Processing, 2003.

[10] D. Pissarenko, Eigenface-based, Facial Recognition. Pp. 1-6, 2002. 